\title{
A Practical Approach to Spectrum Analyzing Unit Using RTL-SDR
}

Md. Habibur Rahman and Md. Mamunoor Islam*

\author{
Department of Electrical and Electronic Engineering, Chittagong University of \\ Engineering and Technology, Chittagong, Bangladesh.
}

*Corresponding author: mcm.mithu@gmail.com

\begin{abstract}
In the present scenario, there has been an immense advancement in the field of wireless communication in this modern engineering world. Now-a-days Software Defined Radio (SDR) technology is an indisputable emerging technology and presents new challenges for communications engineers. The advancement of SDR system has made significant progress in recent years which makes it as a serious substitute of traditional hardware radio architectures where the mathematical procedures are obligatory to decode and process radio signals using analogue circuitry. Recently, computers have turned out to be powerful enough to do the required mathematical calculations using software. So aim of this paper is to demonstrate a RTL-SDR based spectrum analyzer which can be used proficiently as an alternative of existing hardware spectrum analyzer. This approach will lessen the complexity of analogue hardware system with the higher tractability of software based filtering and demodulation techniques. As RTL-SDR devices are quite cheap (Approximately 20\$) and small sized, this system also offers cost effectiveness with provision of portability. An experimental study was conducted with suitable conditions to examine the feasibility and efficiency of the proposed system. The outcome of experimental result is thoroughly examined in this paper.
\end{abstract}

Keywords: Software defined radio (SDR); RTL-SDR dongle; Spectrum analyzer; Tuner IC; Waterfall display.

\section{INTRODUCTION}

Traditionally spectrum analyzing activities are mainly performed by governmental agencies where expensive specialized hardware setups are used [1]. With these radio architectures, a special receiver for almost each radio communication standard is needed. Moreover in many devices, the radio hardware and the decoder hardware are amalgamated on the same board or are not intended to work individually. As a result, using existing radio hardware for an unintended purpose turns out to be problematic or even impossible, which means high costs for specified radio hardware, e.g. for research purposes [2].

In recent years, SDR technology has turn out to be a revolution by bringing much functionality as software with the reduction of the cost of hardware maintenance and upgradation [3]. It is an extremely low-priced software defined radio based on DVB-T TV (Digital HD TV) USB receiving dongles which has RTL2832U chip in it. In March 2010, Eric Fry, Antti Palosaari and the Osmocom team first discovered this device that was developing their own SDR at that time [4]. From then, several approaches have been made by the researchers all over the world employing this device on their research works. In 2014, software-defined sensor architecture for large-scale wideband spectrum monitoring system has been proposed (Damian Pfammatter (et al.) where distributed data collections have been done in real-time over the Internet using RTL-SDR [5]. Another 
approach has been made by Ken Tapping et al. who have presented SDR technology as an alternative to switched radiometers for continuum radio astronomy [6]. A User-Friendly Android-Based Tool for Spectrum-Analysis based on RTL-SDR has been approached by Jens Saalmüller et al. too [7]. In early 2015, a concept of wireless spectrum analyzer in pocket has also been developed using RTL-SDR (Tan Zhang et al.) [8].

One of the main features of RTL-SDR is the transfer of a complete signal spectrum in a selected frequency range with a defined sample rate to the computer. This means that all received data is available in a raw format and can be used without the restrictions and information losses of traditional radio hardware, e.g. caused by a fixed filter bandwidth or signal demodulation. Therefore, one single device can work as a receiver for very different types of signals. This leads to advanced radios that previously required complicated analogue hardware now being able to be implemented easily in software [2]. So in this paper, RTL-SDR has been demonstrated as an alternative approach of a spectrum analyzing unit with advanced radio capabilities such as wideband tuning and waterfall displays.

\section{SYSTEM ARCHITECTURE}

The projected system is based on the RTL-SDR device, a multi-purpose wide band radio scanning unit consisting of economical hardware entity for signal reception and a software portion for signal processing. The hardware part which is available in the form of DVB-T USB dongle, consists of an antenna connected to a tuner chip which is connected to the RTL2832U chip via I2C [7]. The tuner IC has been used for receiving the analog signal and filtering out the desired frequency. Then it transforms this frequency down to an intermediate frequency (IF) generating in-phase and quadrature components (I/Q signals) and sending them into the RTL2832U chip. This chip then samples the signal with a maximum sampling rate of $3.2 \mathrm{MS} / \mathrm{s}$ with 8 bit I/Q samples output. These samples are then sent to the computer via USB. The software part finally processes the raw samples data and illustrates the signal spectrum with waterfall display. Figure 1 illustrates the basic operational method of the whole proposed system.

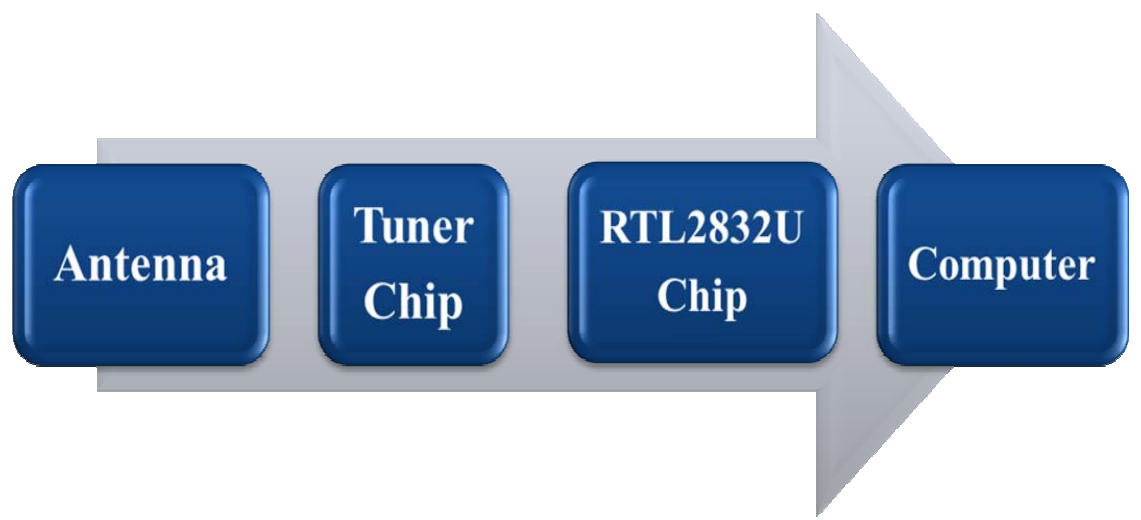

Fig. 1. Basic Oparational Method 


\section{A. The RTL2832U Demodulator}

The RTL2832U is a baseband demodulator which is precisely designed for receiving DVB-T and radio broadcasting. But the application of this demodulator is not limited to these operations. The RTL2832U supports Zero-IF and low IF frequency and has a maximum sample rate of 3.2 MS/s. It receives the IF I/Q signals from the analog tuner IC and outputs the 8 bit I/Q samples [7]. The RTL2832U contains ADC (Analog-to-Digital Converter) and DSP (Digital Signal Processor). It performs DDC (Digital DownConversion) via I/Q mixers (phase is 90 degrees apart), digital low-pass filtering, me /Q resampling, and sends 8-bit I/Q data via the USB port [9]. The RTL2832U contains USB 2.0 interface which supports full and high speed modes. This interface has been used to transfer the samples via bulk transfer to the connected host and also to configure the chip through control transfer messages. Another feature of this interface is that it can act as a repeater for the I2C bus. If the repeater is enabled, control messages which are received over USB are forwarded to the I2C bus as well as messages received on the I2C bus are forwarded to the USB port. This mode permits configuration of the tuner chip through USB interface, as the tuner chip is connected to the RTL2832U via the I2C interface.

\section{B. Tuner Chip}

Almost any DVB-T dongle with the RTL2832U chip can be used with the RTL-SDR drivers. However, one must pay attention to the tuner chip used in the dongle. The tuner chip defines the frequency range of the dongle.

TABLE I. FREQUENCY RANGE OF TUNER CHIPS.

\begin{tabular}{|l|l|l|}
\hline Tuner & $\begin{array}{l}\text { Minimum Frequency } \\
(\mathbf{M H z})\end{array}$ & $\begin{array}{l}\text { Maximum Frequency } \\
(\mathbf{M H z})\end{array}$ \\
\hline R820T & 24 & 1766 \\
\hline E4000 & 52 & 2200 \\
\hline FC0012 & 22 & 948.6 \\
\hline FC0013 & 22 & 1100 \\
\hline
\end{tabular}

There are two commonly used tuners such as R820T and E4000 chips. There are also the less common FC0013 and FC0012. Recently there is also the R828D and FC2580 which are even less common.

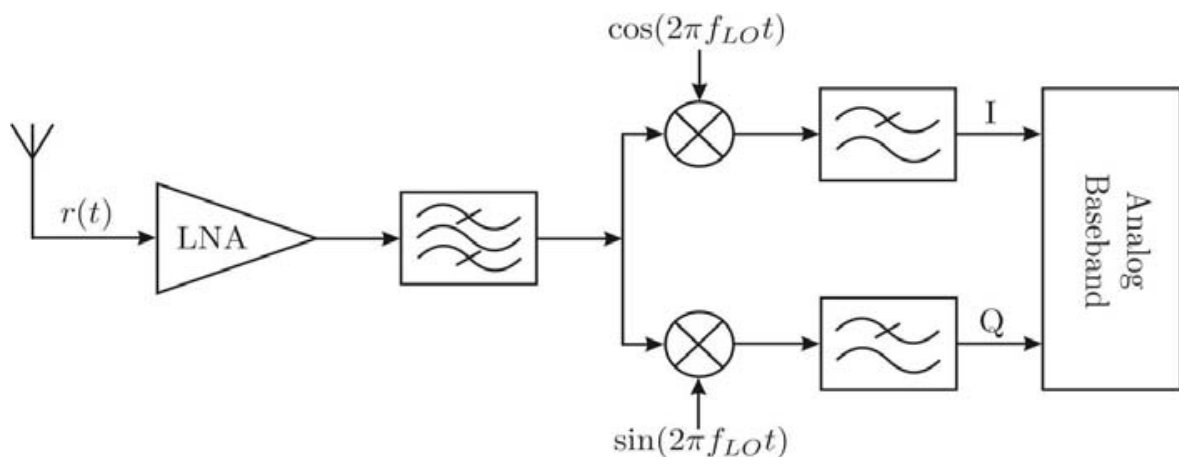

Fig. 2. General signal processing inside a tuner IC [7] 
Each tuner offers different frequency ranges, gains, amplifiers and filters. But they support a frequency range of at least 60 to $1100 \mathrm{MHz}$ in common which is very wide spread range. Figure 2 illustrates an overview of the signal processing inside the tuner IC. At first, the received RF signal is passed into a low-noise amplifier (LNA) where the signal is amplified either automatically or by a manually configurable gain. Next, a certain frequency range is filtered out according to the selected frequency band (VHF II, VHF III, UHF or L-band). After that the mixer transforms the signal into a low frequency IF or Zero-IF and transfers it to the intermediary frequency filter section and gain section where the frequency range is narrowed down to extract the preferred frequency and bandwidth [7].

\section{Software}

More than hundreds software are used on RTL SDR for different purpose in different platform [10]. The most commonly use and available package are given below.

TABLE II. DIFFERENT TYPES OF SOFTWARE.

\begin{tabular}{|l|l|l|l|c|}
\hline \multicolumn{2}{|l|}{ Windows based } & Linux based & \multicolumn{2}{l|}{ Android-Based } \\
\hline Free & Paid/Trail & Free & Free & Paid/Trail \\
\hline SDR\# & Matlab & GNU Radio & RFAnalyzer & SDR Touch \\
\hline HDSDR & Sodira & Linrad & & Wavesink Plus \\
\hline Linrad & Studio1 & GQRX & & \\
\hline CubicSDR & & QtRadio & & \\
\hline QtRadio & & ShinySDR & & \\
\hline SeeDer & & & & \\
\hline
\end{tabular}

From those packages, SDR\# is chosen for several facilities. At present it is the most popular windows based free RTL-SDR compatible software. Set up procedure is relatively easy with respect to other one [11]. It has abundant amount of GUI which make it easy to use. It has some advanced features such as different plugins. Though most of plugins are in $3^{\text {rd }}$ party, those are effective. MATLAB also released the RTL SDR plugging on their R2013b version [12]. With this support package, MATLAB can interface with the RTL-SDR and digital signal processing algorithm can then be written in MATLAB. GNU Radio is another powerful tool for SDR technology [13]. But complexity may arise at the time of installation. Both software are most powerful in research sector. In recent version of LAB View, there have also scope for interfacing with the RTL-SDR.

\section{IMPLEMENTATION AND RESUlt ANALySIS}

A. GSM Signal

The GSM signal has also been received by this spectrum analyzing system. In Bangladesh, GSM 900 and GSM 1800 bands have been used for mobile communication [14]. Banglalink, a local mobile operator in Bangladesh uses 895.2-900.2 MHz frequency band for uplink and 940.2-945.2 MHz frequency band for downlink [14]. This uplink and downlink frequencies received by RTL-SDR (both in windows and Linux Platform) are illustrated in Figure 3, 4 and 5. 


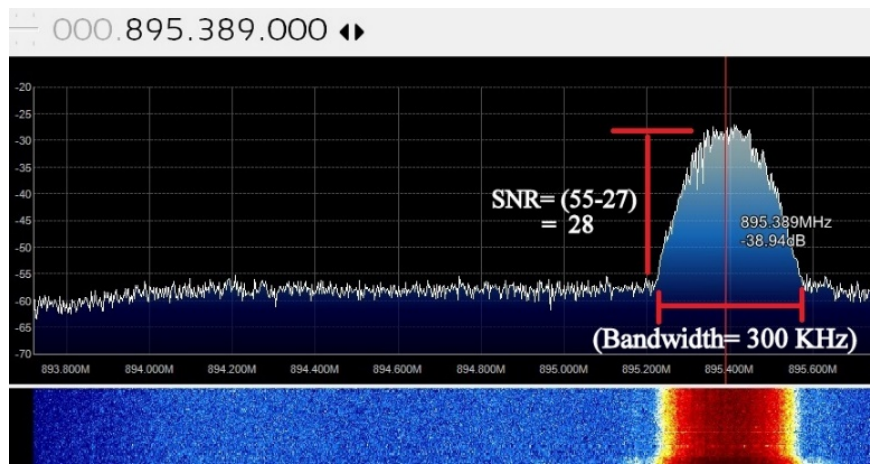

Fig. 3. FFT Spectrum of Uplink Frequency with Waterfall Display in windows platform (SDR\#)

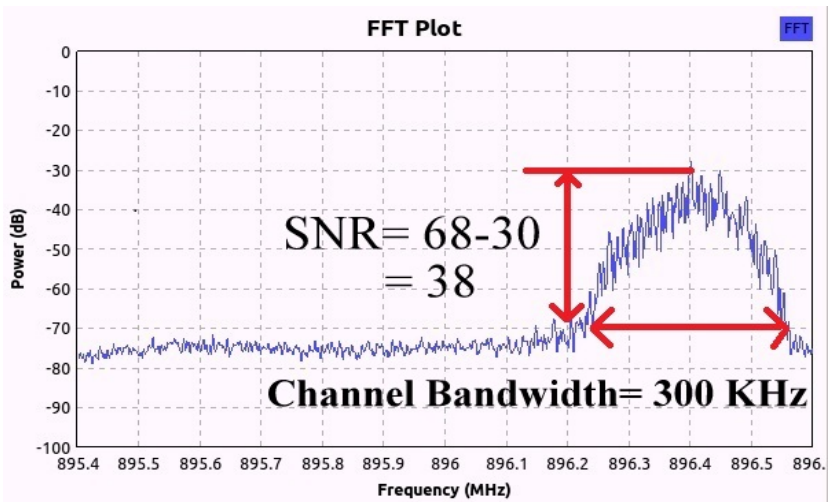

Fig. 4. FFT Spectrum of Uplink Frequency in Linux Platform (GNU Radio)

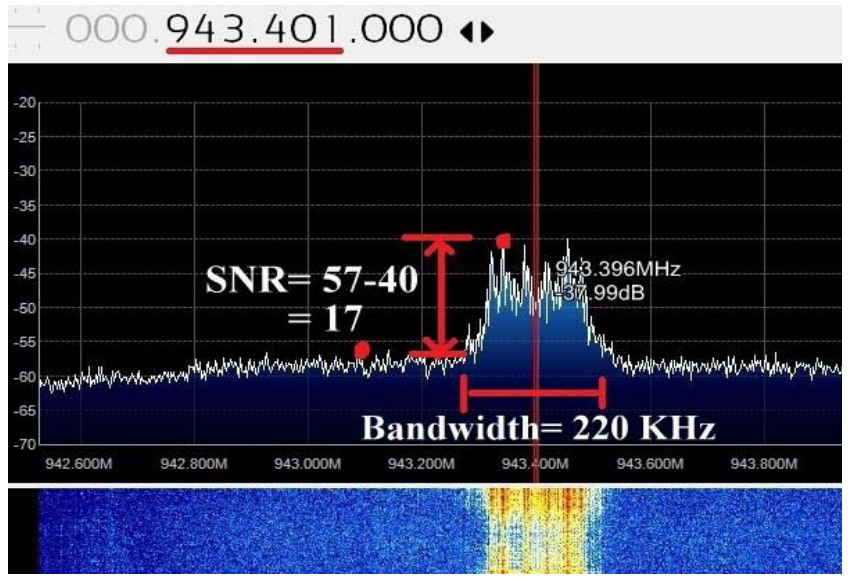

Fig. 5. FFT Spectrum of Downlink Frequency in windows platform (SDR\#) 


\section{B. ISM Band}

The ISM bands, known as industrial, scientific and medical bands are the radio bands which are reserved internationally for the purpose of industrial, scientific and medical uses [15]. Generally communication equipment functioning in these bands has to endure any interference generated by ISM equipment, and users have no regulatory shield from ISM device activities.

As there are numerous allocations, use of these bands has been increasing enormously for short-range and low power communications systems in recent years. Cordless phones, Bluetooth devices, near field communication (NFC) devices and wireless computer networks all use frequencies allocated for ISM bands [15].

TABLE III. ALLOCATIONS OF DIFFERENT ISM BANDS

\begin{tabular}{|l|l|l|}
\hline Frequency Range (MHz) & $\begin{array}{l}\text { Bandwidth } \\
(\mathbf{K H z})\end{array}$ & Availability \\
\hline $6.765-6.795$ & 30 & Subject to local acceptance \\
\hline $13.553-13.567$ & 14 & Worldwide \\
\hline $26.957-27.283$ & 326 & Worldwide \\
\hline $40.660-40.700$ & 40 & Worldwide \\
\hline $433.050-434.790$ & 1740 & Subject to local acceptance \\
\hline
\end{tabular}

In this experimental approach, the ISM band $434 \mathrm{MHz}$ has been tested as an example by a RF frequency generator and the signal has been received by the RTL-SDR system which is illustrated in Figure 6 below.

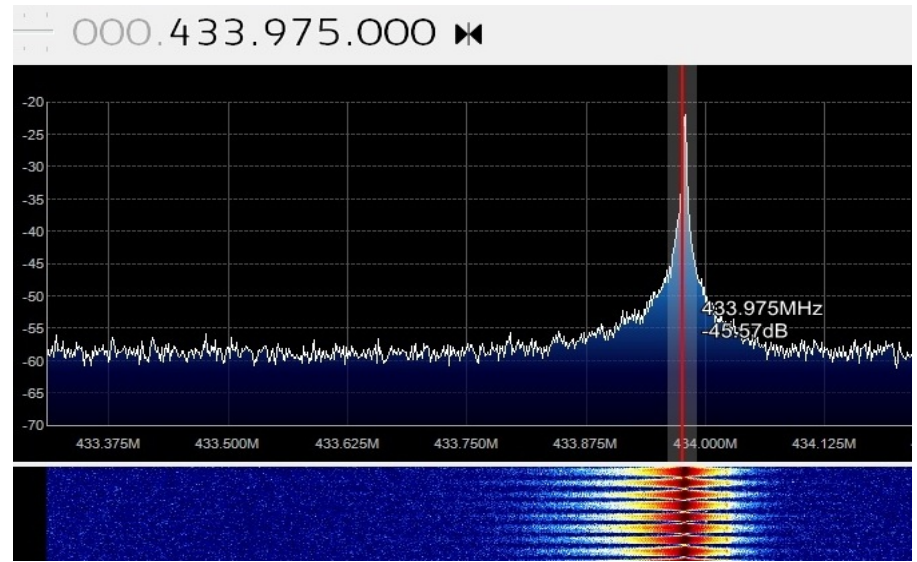

Fig. 6. FFT Spectrum and Waterfall Display of ISM Band Frequency

\section{APRS Signal}

APRS (Automatic Position Reporting System) is a packet based system used to transmit data like messages, announcements weather station data and also to report GPS coordinates of things like transmitters and moving vehicles [4]. APRS frequency is set to Narrow-Band Frequency Modulation (NFM). The FFT spectrum of APRS signal has been illustrated in Figure 7. 


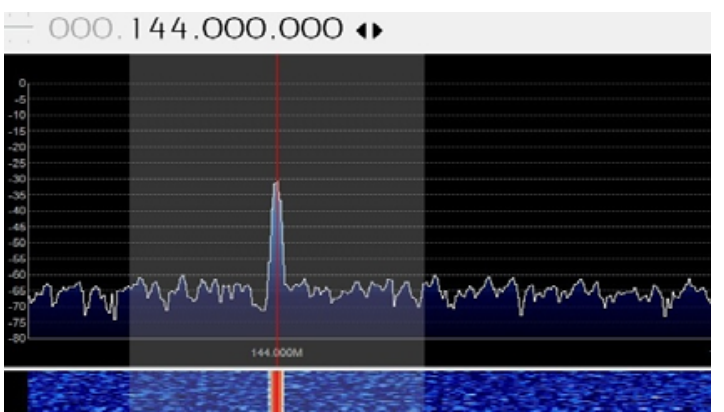

Fig. 7. FFT Spectrum of APRS Signal with Waterfall Display

\section{TV Broadcasting Signal}

A local TV signal of Bangladesh at $172.8 \mathrm{MHz}$ is received by the RTL-SDR system too. The FFT spectrum of this signal is displayed at Figure 8.

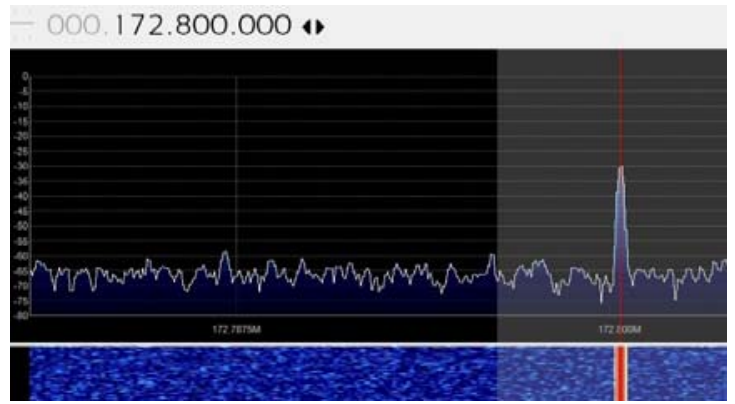

Fig. 8. FFT Spectrum of TV Broadcasting Signal with Waterfall Display

\section{E. FM Broadcasting Signal}

FM broadcasting, a VHF broadcasting technology uses frequency modulation (FM) technique to provide high-quality sound over broadcast radio. The FM signal can also be received by this spectrum analyzing unit. A local Bangladeshi FM broadcasting signal at 89.6 $\mathrm{MHz}$ is illustrated in Figure 9 below.

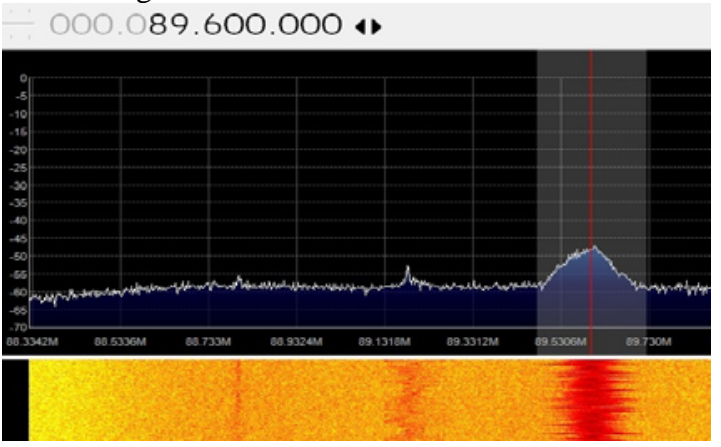

Fig. 9. FFT Spectrum of FM Broadcasting Signal with Waterfall Display 


\section{F. Walkie Talkie}

A walkie-talkie known as a handheld transceiver (HT) is a hand-held, portable and twoway radio transceiver. The FFT spectrum of Walkie Talkie by this RTL-SDR device is illustrated in Figure 10.

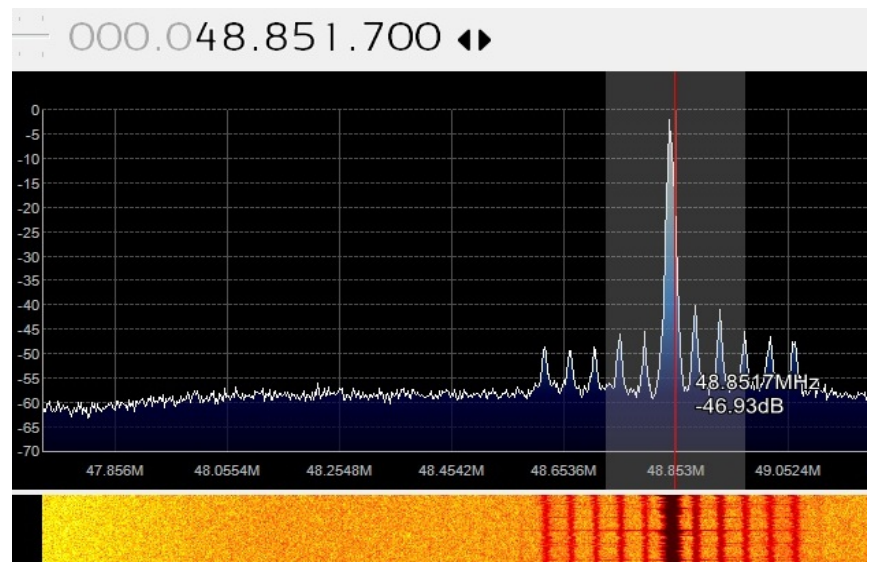

Fig. 10. FFT Spectrum of Walkie Talkie with Waterfall Display

TABLE IV. EXPERIMENTED DATA.

\begin{tabular}{|l|l|l|}
\hline Signal & $\begin{array}{l}\text { Measured Approximate } \\
\text { Bandwidth ( KHz) }\end{array}$ & $\begin{array}{l}\text { SNR } \\
\text { ( Signal to Noise Ratio) }\end{array}$ \\
\hline GSM Uplink & 300 & 28 \\
\hline GSM Downlink & 220 & 17 \\
\hline FM Signal & 210 & 13 \\
\hline Walkie Talkie & 50 & 55 \\
\hline ISM Band & 200 & 33 \\
\hline TV Signal & - & 30 \\
\hline APRS Signal & - & 30 \\
\hline
\end{tabular}

In the Table III, We have experimented some of the signals and measured aprroximate bandwidth and SNR. The experimented data are not accurate but close to the real ones. For TV broadcasting signal, the bandwidth can not be measured beacause the bandwidth exceeds the measuring range of this device. As the signals are received from a place far from base stations, the SNRs are less.

\section{FUTURE WORK}

In future, we make it as a wide band portable spectrum analyzer which may be applied on integrated single board pc. Hence the complexity of using laptop will be removed .Tinny screen will help to see both FFT and waterfall diagram by which SNR and bandwidth will be calculated automatically. To make this device as a vector analyzer is also possible. Decrypting of different types of signal and message is another great scope by this device. 


\section{CONCLUSIONS}

In this paper, it is shown that the RTL-SDR device can be used as an alternative for spectrum analyzing purposes by which the complexity in signal analysis can be lessened easily. Though the performance of this system is not fully apposite, the system is both user-friendly and cost effective compared to the traditional hardware system. So it can be used as a modern spectrum analyzing tool which has wide range of frequency tuning conveniences for analyzing the signal more proficiently.

\section{References}

[1] Ana Nika, Zengbin Zhang, Xia Zhou, Ben Y. Zhao and Haitao Zheng, "Towards Commoditized Real-time Spectrum Monitoring,” HotWireless’14, September 11, 2014, Maui, Hawaii, USA.

[2] Thomas Rudolph, “Analyzing Security-related Signals Using Software defined Radio," Bachelor's Thesis. February 12, 2013.

[3] Govarthanam K S, Abirami M, Kaushik J, "Economical Antenna Reception Design for Software Defined Radio using RTL-SDR,” Proceedings of the Intl. Conf. on Innovative trends in Electronics Communication and Applications 2014, page 185-190.

[4] Carl Laufer, "The Hobbyist's Guide to the RTL-SDR: Really Cheap Software Defined Radio,” Kindle Edition, Published on May 14, 2014.

[5] Damian Pfammatter, Domenico Giustiniano, Vincent Lenders, "A Software-defined Sensor Architecture for Large-scale Wideband Spectrum Monitoring," 14th International Conference on Information Processing in Sensor Networks (IPSN '15), April 14-16, 2015, Seattle, WA, USA.

[6] "Radio Alternative to Switched Radiometers for Continuum Radio Astronomy", www.sbrac.org/files/DTP_RX.pdf (Retrieved on September 2016).

[7] Jens Saalmüller, Matthias Kuba, Andreas Oeder, "A User-Friendly Android-Based Tool for 868 MHz RF Traffic- and Spectrum-Analysis,” embedded world 2015, February 24-26, 2015, Nuremberg, Germany.

[8] Tan Zhang, Ashish Patro, Ning Leng, Suman Banerjee, “A Wireless Spectrum Analyzer in Your Pocket,” HotMobile’15, February 12-13, 2015, Santa Fe, New Mexico, USA.

[9] Dr. Phil, "Realtek RTL2832U: The mystery chip at the heart of RTL-SDR", version 1, published on 2015.

[10] sdr.osmocom.org/trac/wiki/rtl-sdr (Retrieved on September 2016).

[11] uk.groups.yahoo.com/neo/groups/SDRSharp/info (Retrieved on September 2016).

[12] Communications System Toolbox Support Package for RTL-SDR Radio, www.mathworks.com/matlabcentral/fileexchange/44991-communications-system-toolboxsupport-package-for-rtl-sdr-radio (Retrieved on September 2016).

[13] gnuradio.org/redmine/projects/gnuradio (Retrieved on September 2016).

[14] www.spectrummonitoring.com/frequencies/frequencies3.html\#Bangladesh (Retrieved on September 2016).

[15] “ARTICLE 1 - Terms and Definitions,” life.itu.ch. International Telecommunication Union. 19 October 2009. 